\title{
Literacia para a saúde em países de renda baixa ou média: uma revisão sistemática
}

\author{
Health literacy in low- and middle-income countries: \\ a systematic review
}

Ana Luiza Braz Pavão (http://orcid.org/0000-0002-4122-1796) ${ }^{1}$

Guilherme Loureiro Werneck (https://orcid.org/0000-0003-1169-1436) ${ }^{2}$

\footnotetext{
${ }^{1}$ Instituto de Comunicação e Informação Científica

e Tecnológica em Saúde, Fundação Oswaldo Cruz. Av Brasil 4.365 Pavilhão Haity Moussatché, Manguinhos. 21040-900 Rio de Janeiro RJ Brasil. ana.pavao@icict.fiocruz.br ${ }^{2}$ Instituto de Medicina Social, Universidade do Estado do Rio de Janeiro. Rio de Janeiro RJ Brasil.
}

\begin{abstract}
Health literacy $(H L)$ is linked to individual capacities of access, understanding, assessment and application of health information to make decisions in everyday life, in order to maintain or improve health. The scope of this article is to review studies on HL conducted in low-and middle-income countries, with an emphasis on the definition used for HL. It involved a systematic search in the Medline, Embase, Scopus, LILACS and SciELO databases. It included studies that showed the definition of HL, studies in countries with low- and middle-income economies and Latin American studies. Initially, a selection of studies was made by reading the titles and/or abstracts. Two independent evaluators conducted the reading of the full text. Disagreements were discussed by consensus. A total of 6,025 references were located and 36 were selected for the final sample. Most studies (58.3\%) were from countries on the Asian continent, followed by studies from South American countries (27.8\%), including Brazil. Most studies (58.3\%) evaluated the functional dimension of the HL (FHL). The most frequent definitions were from the Institute of $\mathrm{Me}$ dicine and from the World Health Organization. Approximately 30\% of the studies that evaluated FHL used broader definitions of $H L$ as theoretical frameworks.
\end{abstract}

Key words Review, health, literacy, Public health
Resumo Literacia para a Saúde (LS) relacionase às capacidades individuais de acesso, compreensão, avaliação e aplicação das informações de saúde, a fim de se tomar decisões na vida diária, para manter ou melhorar a saúde. O objetivo deste artigo é revisar os estudos sobre LS conduzidos nos países de renda baixa ou média, com ênfase na definição utilizada para LS. Revisão sistemática nas bases de dados Medline, Embase, Scopus, LILACS e SciELO. Foram incluídos estudos que apresentavam a definição de LS, estudos em países de economias de renda baixa ou média e estudos latino-americanos. Inicialmente foi feita leitura dos títulos elou resumos. Dois avaliadores independentes realizaram a leitura do texto completo. Discordâncias foram discutidas por consenso. Foram encontradas 6.025 referências e selecionadas 36 para a amostra final. A maioria dos estudos (58,3\%) era de países do continente asiático, seguidos pelos estudos em países da América do Sul (27,8\%), incluindo o Brasil. A maior parte (58,3\%) avaliou a dimensão funcional da LS (LFS). As definições mais frequentes foram do Instituto de Medicina dos Estados Unidos e da Organização Mundial de Saúde. Aproximadamente $30 \%$ dos estudos que avaliaram a LFS utilizaram como referencial teórico definições mais abrangentes de LS.

Palavras-chave Revisão, Saúde, Alfabetização, Saúde pública 


\section{Introdução}

O conceito de Literacia para a saúde (LS) vem ganhando importância, tanto no âmbito da pesquisa, como da prática clínica e, sobretudo, no campo da saúde pública ${ }^{1}$. Diversos estudos vêm evidenciando sua associação com determinantes sociais em saúde, aspectos comportamentais e desfechos em saúde, uso de serviços de saúde, barreiras de acesso, qualidade dos sistemas de saúde e até mortalidade precoce ${ }^{2-6}$. A Organização Mundial de Saúde (OMS) definiu LS como a representação das habilidades sociais e cognitivas que determinam a motivação e as experiências dos indivíduos em obter acesso, compreender e usar as informações de forma que promovam ou mantenham a sua saúde ${ }^{7}$. Segundo este conceito, LS significa muito mais do que ser capaz de ler rótulos, panfletos ou anotações de saúde. Ao promover o acesso das pessoas à informação sobre saúde e, ao capacitá-las a utilizar essa informação de forma eficiente, torna-se uma ferramenta de empoderamento dos indivíduos ${ }^{78}$.

A pesquisa sobre literacia para a saúde ganhou força nas últimas décadas, sobretudo nos países desenvolvidos, como Estados Unidos da América, Canadá, Austrália, Reino Unido, Japão, Holanda, Suíça ${ }^{9-14}$ e vem sendo considerado tema prioritário em ações de saúde estratégicas, tanto nos Estados Unidos, como na Europa. Apesar de sua importância para a saúde pública, esse tema ainda é pouco explorado em países em desenvolvimento. Tendo em vista a sua associação com diversos desfechos em saúde, quando em situação de baixo nível de literacia, como o menor uso de serviços preventivos, aumento nas barreiras de acesso aos serviços de saúde, aumento nas internações hospitalares, menor adesão a tratamentos, mudanças nos comportamentos de saúde, e até influências nos determinantes sociais de saúde e na mortalidade precoce, considera-se que esse é um campo que merece especial atenção por parte dos pesquisadores da área da saúde, pois, além de ser um importante indicador, é uma ferramenta de empoderamento dos indivíduos em relação a sua saúde ${ }^{15}$.

Como a grande maioria dos estudos sobre LS conduzidos até o momento foram realizados no contexto de países desenvolvidos e, diante da existência de diferentes definições e abordagens para o construto da LS, o objetivo do presente estudo é realizar uma revisão sistemática dos estudos que analisaram a LS nos países de renda baixa ou média, com ênfase na definição conceitual utilizada para o construto da LS nesses países.

\section{Metodologia}

O desenho do presente estudo é o de uma revisão sistemática. Para atingir ao objetivo proposto, foram realizadas duas buscas em cinco bases de dados: Medline (Pubmed), Embase, Scopus, LILACS e SciELO.

As buscas foram realizadas entre julho e setembro de 2017 e a data-limite de inclusão dos estudos foi 31 de julho de 2017. Foram incluídos na busca todos os estudos publicados antes dessa data. Não foram utilizados limites relacionados à idade da população do estudo.

\section{Estratégia de busca}

A primeira busca (BUSCA 1) teve por objetivo captar os estudos que abordassem a definição conceitual de LS. A estratégia utilizada para a base Pubmed encontra-se abaixo:

((Definition OR concept OR conceptual framework OR taxonomy) AND ("health literacy" OR "health competence" OR "health literacy" [MeSH Terms]))

Para as demais bases de dados, a estratégia de busca (BUSCA 1) foi a mesma acima, exceto pela não inclusão do termo MeSH.

A segunda busca (BUSCA 2) teve como objetivo ampliar a captação de estudos conduzidos sobre o tema da LS em países menos desenvolvidos e/ou países latino-americanos e de estudos que utilizassem outras traduções para o termo LS. Utilizou-se a seguinte estratégia de busca, para as bases de dados Pubmed, Embase e SciELO:

"connaissances en matiére de santé" OR "alphabétisme en matiére de santé" OR "littéracie en matiére de santé" OR "littératie en santé" OR "literacia em saúde" OR "educação em saúde" OR "alfabetização em saúde" OR "letramento em saúde" OR "instrucción sanitária” OR "alfabetización en salud" OR "compétence en santé"

Para as demais bases (Scopus e LILACS), a mesma estratégia acima foi utilizada (BUSCA 2), porém foi necessário realizar várias buscas separadamente devido à limitação de espaço no campo de busca dessas bases.

As palavras-chave selecionadas para a construção das estratégias de busca foram baseadas em uma breve revisão feita pela pesquisadora principal de estudos prévios sobre o tema da LS, dentre eles uma revisão sistemática conduzida em países desenvolvidos ${ }^{16}$, além de consulta feita a um especialista na área de LS e promoção de saúde. 
A primeira seleção foi feita pela pesquisadora principal, com base na leitura dos títulos e/ ou resumos. A segunda seleção foi feita por dois avaliadores independentes, com a leitura do texto completo. As discordâncias foram discutidas buscando-se consenso.

O Quadro 1 apresenta as estratégias de busca utilizadas para cada base de dados.

\section{Critérios de seleção}

Foram aplicados critérios de seleção dos estudos, tanto na primeira como na segunda seleção. Em relação a esses critérios, foram utilizados os seguintes critérios de inclusão e exclusão:

\section{Critérios de inclusão}

- Estudos que tratavam do tema da LS e que traziam de forma clara o seu conceito ou definição;

- Estudos conduzidos em países de economias de renda baixa ou média, segundo o critério do Banco Mundial ${ }^{17}$ e estudos latino-americanos.

Segundo a classificação do Banco Mundial, para o ano fiscal de 2018, são considerados países com economias de renda baixa àqueles com PIB per capita igual ou abaixo de U\$ 1.005 em 2016, países com economias de renda média inferior com PIB per capita entre U\$ 1.006 e U\$3.955, países com economias de renda média alta com PIB per capita entre U\$3.956 e U\$ 12.235 e países com economias de renda alta àqueles com PIB per capita igual ou superior a U\$ 12.236.

Além dos estudos conduzidos em países de renda baixa e média, foram incluídos os estudos em países latino-americanos, pois, segundo o critério do Banco Mundial, um desses países foi classificado como renda alta (Chile).

\section{Critérios de exclusão:}

- Estudos que não possuíam resumo e/ou texto completo disponível;

- Estudos cujo texto completo estivesse em outro idioma diferente de: português, espanhol, inglês e francês;

- Estudos de revisão (ex: revisões, revisões sistemáticas ou meta-análises);

- Estudos que não apresentavam de forma clara o conceito de LS utilizado, ou que versavam sobre outro tema, ou estudos em que o foco principal não era a LS ou que citaram muito pouco esta definição;

- Estudos que não se enquadravam na lista de países com economias de renda baixa ou média, segundo o critério do Banco Mundial ${ }^{17}$, exceto se países latino-americanos;

- Estudos repetidos (duplicatas).

A literatura cinzenta também foi incorporada nesta revisão, mediante a consulta a especialistas no tema da LS (nacionais e internacionais) e através da busca em diferentes bases de dados, especialmente Embase e Scopus, que trouxeram publicações oriundas de congressos científicos, dentre outras.

Após a realização da busca, os arquivos gerados nas diferentes bases de dados foram exportados para o software online de gerenciamento de referências Endnote web, o qual foi utilizado no processo de seleção dos estudos (primeira e segunda seleções) e no compartilhamento de referências entre os avaliadores, após a remoção das duplicidades. O software também permitiu a leitura dos resumos e o direcionamento para o texto completo na maioria dos casos.

Os estudos selecionados na revisão foram avaliados em relação às seguintes características: país de origem, ano de publicação, tipo de publi-

Quadro 1. Estratégias de busca utilizadas (BUSCA 1 e BUSCA 2), segundo as bases de dados.

\begin{tabular}{|l|l|}
\hline \multicolumn{1}{|c|}{ Base de dados } & \multicolumn{1}{c|}{ Estratégia de busca } \\
\hline Medline (Pubmed) & $\begin{array}{l}\text { BUSCA 1 } \\
\text { ((Definition OR concept OR conceptual framework OR taxonomy) AND ("health } \\
\text { literacy" OR "health competence" OR "health literacy" [MeSH Terms])) }\end{array}$ \\
\hline $\begin{array}{l}\text { Embase, Scopus, LILACS } \\
\text { e SciELO }\end{array}$ & $\begin{array}{l}\text { BUSCA 1 } \\
\text { ((Definition OR concept OR conceptual framework OR taxonomy) AND ("health } \\
\text { literacy" OR "health competence" }\end{array}$ \\
\hline $\begin{array}{l}\text { Medline (Pubmed), } \\
\text { Embase, Scopus, LILACS } \\
\text { e SciELO }\end{array}$ & $\begin{array}{l}\text { BUSCA 2 } \\
\text { "connaissances en matiére de santé" OR "alphabétisme en matiére de santé" OR } \\
\text { "littéracie en matiére de santé" OR "littératie en santé" OR "literacia em saúde" OR } \\
\text { "educação em saúde" OR "alfabetização em saúde" OR "letramento em saúde" OR } \\
\text { "instrucción sanitária" OR "alfabetización en salud" OR "compétence en santé" }\end{array}$ \\
\hline
\end{tabular}

Fonte: Elaborado pelos autores. 
cação, desenho do estudo, questionário de avaliação da LS utilizado, dimensão da LS avaliada e definição utilizada para LS no estudo (referencial teórico).

Após a realização das buscas 1 e 2 nas bases de dados Pubmed, Embase, Scopus, LILACS e SciELO, foi encontrado um total de 6.025 referências. Destas, 560 (9,3\%) eram oriundas do Pubmed, 3.743 (62,1\%) do Scopus, 821 (13,6\%) da base Embase, $114(1,9 \%)$ do SciELO e $787(13,1 \%)$ do LILACS (Quadro 2).

Inicialmente, foram removidas, no software Endnote web, 327 duplicidades, restando-se 5.698 referências para avaliação na primeira seleção. Após a aplicação dos critérios de seleção pela pesquisadora principal, foram selecionadas para a segunda fase 257 referências. Ou seja, foram excluídas 5.441 referências na primeira seleção, pela análise do título e/ou resumo. Na segunda etapa da seleção, as duas avaliadoras independentes selecionaram, um total de 36 estudos que atendiam os critérios de seleção. Houve oito divergências que foram dirimidas por consenso. Das 257 referências, portanto, 221 ainda foram eliminadas após a aplicação dos critérios de seleção e leitura do texto completo pelas duas avaliadoras independentes, pelos seguintes motivos: 131 estudos por terem sido conduzidos no contexto de países de renda alta, ou seja, fora da lista do Banco Mundial; 52 por não apresentarem de forma clara o conceito de LS utilizado ou por não terem como foco principal do estudo a temática da LS; 23 por se tratarem de estudos de revisão; 11 por serem estudos cujo texto completo se encontrava em idioma diferente de português, inglês, espanhol ou francês; e quatro por serem estudos repetidos. O fluxograma, a seguir, descreve as etapas de seleção e o número de estudos excluídos e incluídos, até a amostra final de 36 estudos selecionados ${ }^{18-53}$. (Figura 1).

Quadro 2. Total de referências encontradas nas buscas 1 e 2 , segundo as bases de dados.

\begin{tabular}{|l|c|}
\hline \multicolumn{1}{|c|}{ Bases de dados } & $\begin{array}{c}\text { Total de referências } \\
\text { encontradas } \\
\text { (BUSCA 1 + BUSCA 2) - n } \\
(\mathbf{\%})\end{array}$ \\
\hline MEDLINE (Pubmed) & $560(9,3 \%)$ \\
\hline Scopus & $3.743(62,1 \%)$ \\
\hline Embase & $821(13,6 \%)$ \\
\hline LILACS & $787(13,1 \%)$ \\
\hline SciELO & $114(1,9 \%)$ \\
\hline Total & $6.025(100 \%)$ \\
\hline
\end{tabular}

Fonte: Elaborado pelos autores.

\section{Resultados}

\section{Características gerais dos estudos selecionados}

Dos 36 estudos selecionados para a amostra final, nove $(25 \%)$ eram provenientes da China, oito $(22,2 \%)$ de Taiwan, sete $(19,4 \%)$ do Brasil, três $(8,3 \%)$ do Irã, dois $(5,6 \%)$ da Argentina, dois $(5,6 \%)$ da Turquia, dois $(5,6 \%)$ da Sérvia, um $(2,8 \%)$ do Chile, um $(2,8 \%)$ da Tailândia e um $(2,8 \%)$ das Ilhas Marshall. Ou seja, mais da metade dos estudos, $21(58,3 \%)$, eram provenientes de países do continente asiático, dez $(27,8 \%)$ estudos eram provenientes de países da América do Sul, quatro $(11,1 \%)$ eram oriundos de países europeus e um $(2,8 \%)$ da Oceania. Os estudos foram publicados entre os anos de 2009 e 2017. O ano de 2017 foi o que apresentou maior número de publicações, com dez publicações no total $(27,8 \%)$. Em seguida, vieram os anos de 2016, 2015 e 2012, com cinco publicações cada

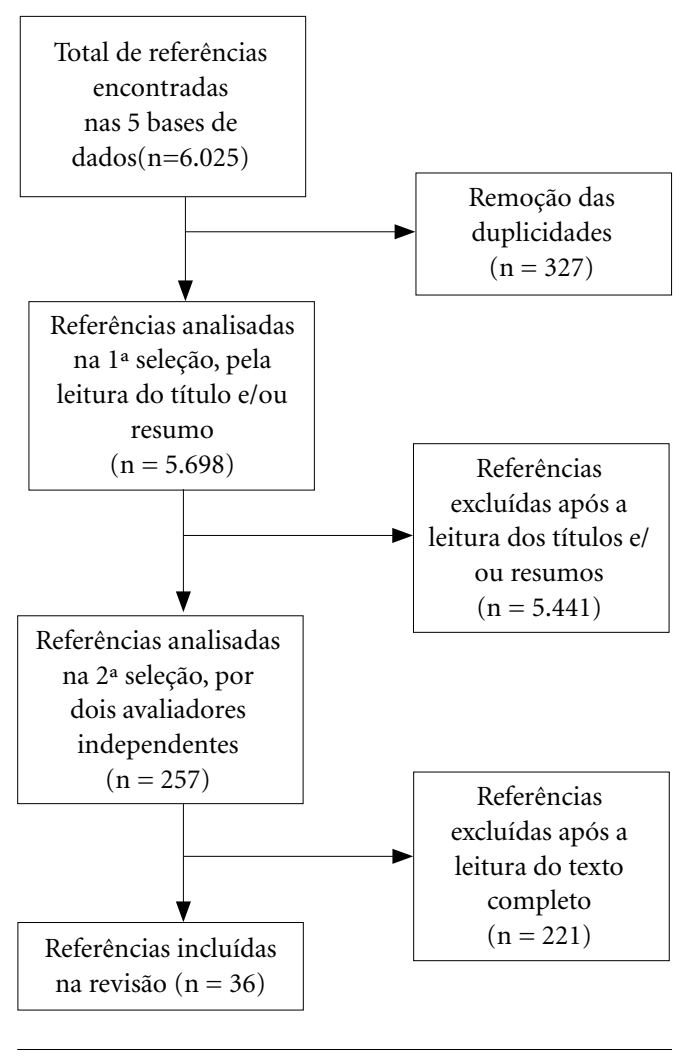

Figura 1. Fluxograma do processo de seleção dos estudos.

Fonte: Elaborado pelos autores. 
(13,9\%), seguidos pelos anos de 2013 e 2014 com 4 publicações cada $(11,1 \%)$. Os anos de 2009, 2010 e 2011 tiveram uma publicação cada $(2,8 \%)$.

Em relação ao tipo de publicação, somente um estudo era um resumo de congresso científico. Os demais $(35 ; 97,2 \%)$ eram artigos originais. Em relação ao desenho de estudo, a maioria dos estudos $(16 ; 44,4 \%)$ eram estudos transversais que envolviam a aplicação de algum instrumento de aferição. Nove estudos eram de validação de questionários (25\%) e sete $(19,4 \%)$ descreviam os processos de desenvolvimento e validação de instrumentos de aferição. Entre os nove estudos sobre validação de questionários, um também envolveu a tradução do instrumento. Dois estudos $(5,6 \%)$ eram descritivos: um relatava as etapas relacionadas ao desenvolvimento de um questionário e o outro consistia no relato sobre o desenvolvimento de uma cartilha educativa que serviu de base para a elaboração de um questionário. Outros dois estudos (5,6\%) eram estudos qualitativos, que também envolviam a realização de entrevistas com os participantes do estudo.

Dos 36 estudos selecionados na amostra final, 35 (97,2\%) envolviam o desenvolvimento, a validação ou a aplicação de algum questionário. Somente um $(2,8 \%)$ estudo descrevia o desenvolvimento de um material educativo, uma cartilha educativa para indivíduos portadores de doença renal crônica. Em cinco dos 35 estudos (14,3\%), o instrumento era o Test of Functional Health Literacy (TOFHLA), em sua versão original ou abreviada; em outros cinco (14,3\%), o instrumento era o Short Assessment of Health Literacy for Spanish Speaking Adults (SAHLSA) ou o Short Assessment of Health Literacy for Portuguese Speaking Adults (SAHLPA), instrumentos derivados do Rapid Estimate of Adult Literacy in Medicine $(\text { REALM })^{69}$. Três estudos $(8,6 \%)$ avaliaram o Newest Vital Sign (NVS) ou o REALM e um estudo $(2,9 \%)$ avaliou o Short-Form Health Literacy Questionnaire (HL-SF12), derivado do European Health Literacy Assessment tool (HLS-EU-Q). Os demais estudos $(21 ; 60 \%)$ envolviam questionários voltados para populações específicas, como adolescentes (dois; 5,7\%), pacientes diabéticos (dois; $5,7 \%$ ) ou com infarto agudo do miocárdio (um; 2,9\%), estudos sobre temas específicos, como doenças infecciosas (três; 8,6\%), odontologia ou saúde bucal (três; 8,6\%), alimentação ou nutrição (um; 2,9\%) e estudos que utilizaram outras escalas de avaliação da literacia relacionada à saúde, elaborada pelos autores, por órgãos governamentais dos seus países de origem ou ba- seadas em escalas publicadas em estudos prévios (nove; 25,7\%).

O Quadro 3 ao final da seção apresenta as características gerais dos estudos selecionados.

\section{Dimensão da Literacia avaliada nos estudos e as principais definições}

Nutbeam, em sua publicação de $2000^{7}$, propôs três dimensões para a LS: a funcional ou básica, a interativa ou comunicativa e a crítica. A LS funcional ou básica diz respeito às habilidades básicas de leitura e escrita, que capacitam os indivíduos para lidarem de forma efetiva nas diferentes situações do dia a dia. A LS interativa ou comunicativa refere-se a habilidades cognitivas e de literacia mais avançadas que podem ser usadas pelos indivíduos para participar ativamente nas atividades diárias, extrair informações e derivar significado das diferentes formas de comunicação e aplicar novas informações para mudar as circunstâncias. A LS crítica, por sua vez, representa as habilidades cognitivas mais avançadas que podem ser aplicadas para analisar informações e usá-las para exercer maior controle sobre diferentes eventos e situações da vida.

Partindo-se dessa avaliação multidimensional do construto da LS, proposta por Nutbeam, os 36 estudos foram analisados com relação ao domínio da LS que foi abordado. Os estudos que se basearam na primeira dimensão de LS proposta por Nutbeam, a LS funcional ou básica, ou simplesmente Literacia Funcional em Saúde (LFS), foram aqueles que majoritariamente utilizaram instrumentos de aferição de habilidades individuais relacionadas à leitura, escrita, compreensão da linguagem escrita e numeramento, tais como: o REALM, o NVS, o TOFHLA, o SAHLSA, o SAHLPA, o Short-Form health literacy scale (SHEAL), o Mandarin Health Literacy Scale (MHLS), o Teste de Avaliação do Letramento em Saúde (TALES) e um instrumento elaborado para avaliar a LFS no contexto de doenças infecciosas respiratórias. No total, 18 (50\%) estudos avaliaram a LFS, ou seja, a primeira dimensão da LS (funcional ou básica).

Dos 18 estudos restantes, 14 (38,9\%) avaliaram o a LS propriamente dita, ou seja, foram capazes, a partir da metodologia utilizada, de ampliar o conceito da LS para as outras dimensões abordadas por Nutbeam (a interativa ou comunicativa e a crítica), conforme mostra a quinta coluna do Quadro 3. Os quatro estudos remanescentes $(11,1 \%)$ tratavam do conceito da literacia em situações específicas, como na odontologia 
ou saúde bucal e a literacia relacionada à alimentação ou nutrição. Os três estudos que avaliavam a literacia oral em saúde ou a literacia aplicada à odontologia utilizavam o conceito da LFS, ou seja, a primeira dimensão da LS. Já o estudo que tratou da literacia relacionada à alimentação ou nutrição, captou o construto multidimensional da LS propriamente dita. Ao contabilizarmos esses estudos, o percentual de estudos que se concentrou na dimensão funcional ou básica da LS seria de $58,3 \%(\mathrm{~N}=21)$, enquanto que o percentual de estudos que conseguiu capturar as demais dimensões da LS aumentaria para 41,7\% ( $\mathrm{N}=15)$.

Em relação ao referencial teórico ou à definição utilizada pelos autores para o construto da LS, nos 36 estudos selecionados para a revisão sistemática, a maioria $(13 ; 36,1 \%)$ utilizou a definição do Instituto de Medicina dos Estados Unidos (IOM), de 2004, segundo a qual LS é definida como o grau com que indivíduos possuem a capacidade de obter, processar e compreender informações básicas sobre saúde, bem como os serviços necessários para se tomar decisões apropriadas em saúde $e^{54}$. Em segundo lugar (sete; $19,4 \%$ ), veio a definição da OMS ${ }^{55}$, de 1998, que define LS como as habilidades cognitivas e sociais que determinam a motivação e a capacidade de os indivíduos obterem acesso a, compreenderem e usarem informações de forma a promover e manter uma boa saúde. Em seguida, esteve a definição proposta por Sorensen ${ }^{16}$, citada em cinco estudos (13,9\%), segundo a qual LS representa o conhecimento, a motivação e as competências dos indivíduos para acessarem, compreenderem, avaliarem e aplicarem as informações sobre saúde, a fim de fazer julgamentos e tomar decisões na vida diária, relacionadas aos cuidados de saúde, à prevenção de doenças e à promoção de saúde, para manter ou melhorar a sua qualidade de vida durante o curso da vida. As definições da Associação Médica Americana $(1999)^{56}$ e dos estudos de Nutbeam ${ }^{2,7}$ foram mencionadas por dois estudos cada $(5,6 \%)$. Houve um estudo $(2,8 \%)$ que mencionou a definição do estudo de Kwan ${ }^{57}$ para LS, segundo a qual é o grau com que ou as habilidades individuais para acessar, compreender, avaliar e comunicar a informação de modo a promover, manter e melhorar a saúde em vários cenários ao longo do curso da vida. Em seis estudos (16,7\%) houve mais de uma referência citada para a definição de LS.

Ao considerarmos o total de citações por referencial teórico para as definições de LS usadas nos 36 estudos (sexta coluna do Quadro 3), houve 41 citações, ao serem incluídos também os estudos que citaram mais de uma referência para a definição de LS. Em ordem decrescente, portanto, considerando o total de citações, aquelas mais frequentes foram: definição do IOM (2004) - 16 citações (39\%), da OMS (1998) - dez citações $(24,4 \%)$, a do estudo de Sorensen (2012) - seis citações (14,6\%), dos estudos de Nutbeam - quatro citações $(9,8 \%)$ da Associação Médica Americana (AMA, 1999) e do estudo de Kwan (2006) - duas citações cada (4,9\%) e do estudo de Parker $(1995)^{58}$ - uma citação $(2,4 \%)$.

Observa-se que, em seis estudos $(28,6 \%)$, dos 21 que avaliaram a dimensão funcional da LS (LFS), incluindo os que analisaram a LFS no contexto da saúde bucal, os autores descreveram como definições para LS àquelas mais relacionadas à multidimensionalidade deste construto, como as definições de: Sorensen ${ }^{16}, \mathrm{OMS}^{55}, \mathrm{Kwan}^{57}$ e Nutbeam ${ }^{2,7}$. Os demais estudos que avaliaram a LFS descreveram mais comumente a definição do IOM para LS ${ }^{55}(11 ; 52,4 \%)$, ou ainda, com menor frequência, a definição da $\mathrm{AMA}^{56} \mathrm{e}$ a de Parker $^{58}$. Por outro lado, entre os 15 estudos que avaliaram o construto da LS em suas múltiplas dimensões, os autores mais frequentemente relataram (14; 93,3\%) as definições dos estudos de Sorensen ${ }^{16}$, $\mathrm{OMS}^{55}, \mathrm{Kwan}^{57}$ e Nutbeam ${ }^{2,7}$. Apenas um estudo deste tipo $^{34}(6,7 \%)$ citou a definição do IOM.

Ainda em relação às definições utilizadas para LS, mais precisamente sobre a nomenclatura utilizada, observa-se que, entre os dez estudos conduzidos em países latino-americanos (Brasil, Argentina e Chile), que utilizam o idioma português ou espanhol, utilizaram-se as seguintes terminologias para representar o construto da LS ou da LFS: alfabetização em saúde (ou "alfabetización en salud") nos dois estudos conduzidos na Argentina, no único estudo conduzido no Chile e em um estudo brasileiro (quatro; 40\%); alfabetismo em saúde em um estudo brasileiro (10\%); letramento em saúde, em quatro estudos brasileiros (40\%) e literacia em saúde em um estudo brasileiro $(10 \%)$. Os demais estudos utilizaram os termos em inglês "health literacy", em sua maioria, "functional health literacy", conforme o caso. Ao considerarmos apenas os sete estudos conduzidos no Brasil, a maioria (quatro; $57,1 \%$ ) utilizou o termo "letramento em saúde" e os três estudos restantes utilizaram termos diferentes entre si, como: alfabetização em saúde, alfabetismo em saúde e literacia em saúde (um;14,3\% cada um, respectivamente).

O Quadro 3, a seguir, apresenta os referenciais teóricos (definições) e as nomenclaturas utilizadas em cada um dos 36 estudos selecionados para revisão. 


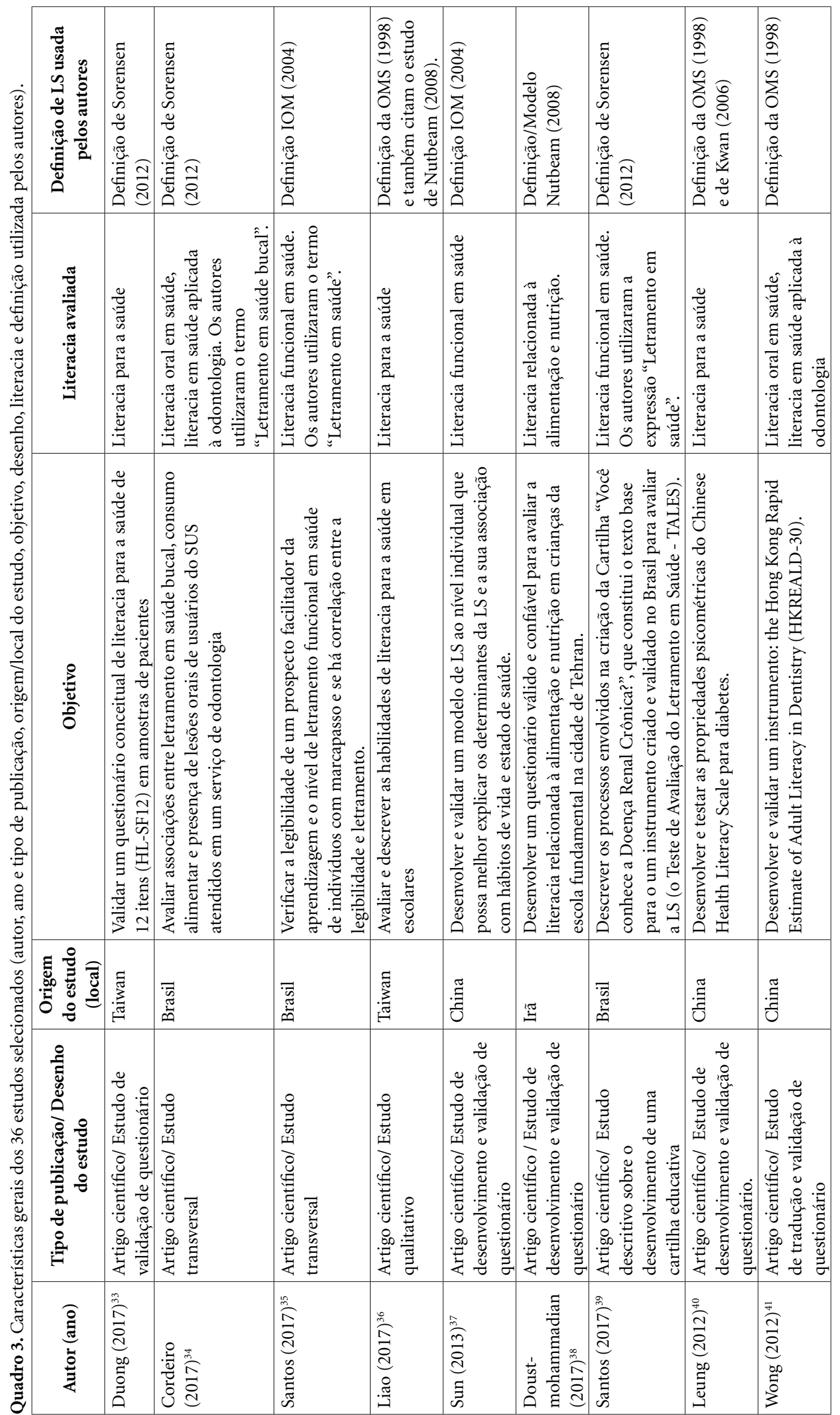




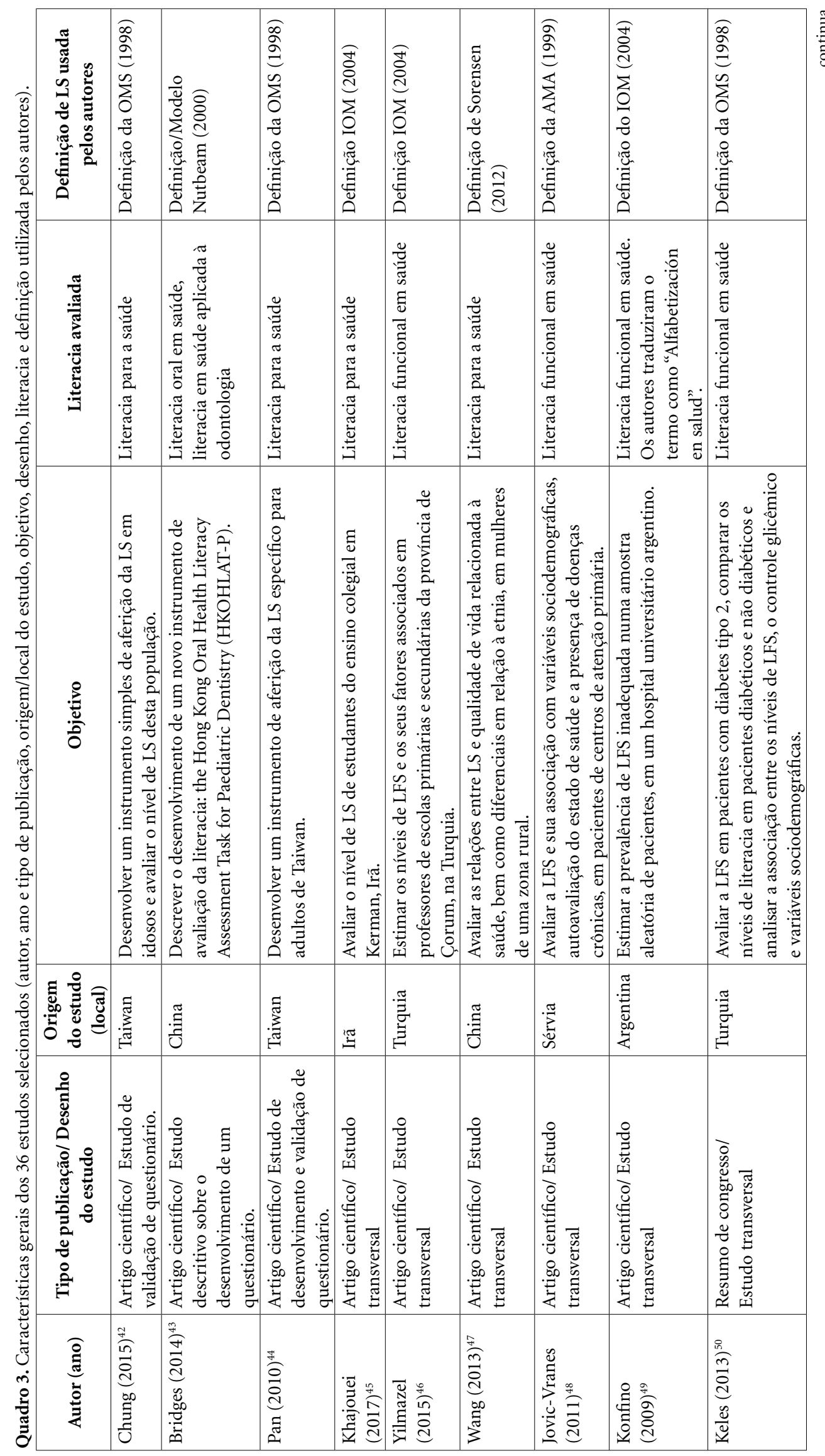




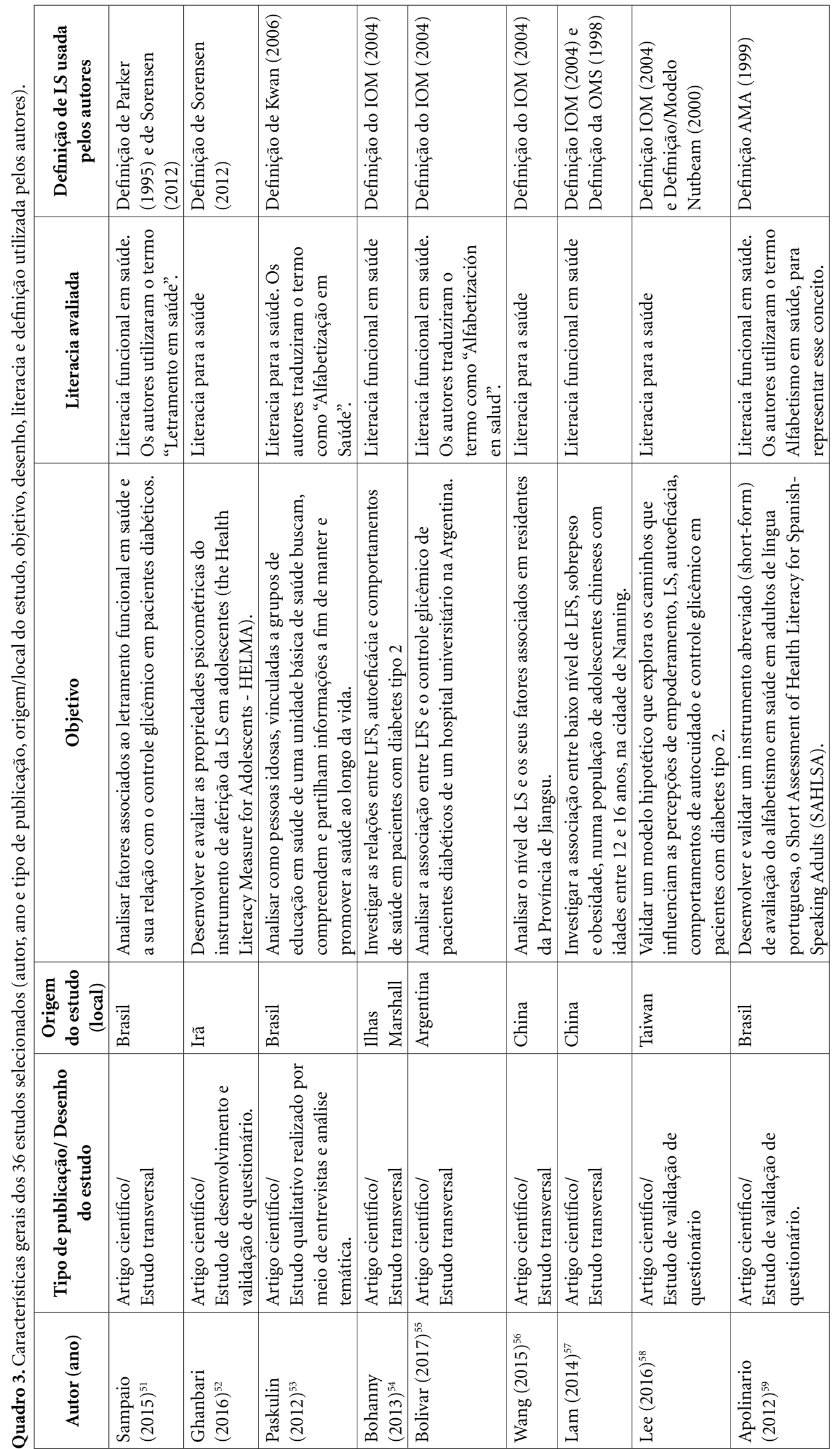




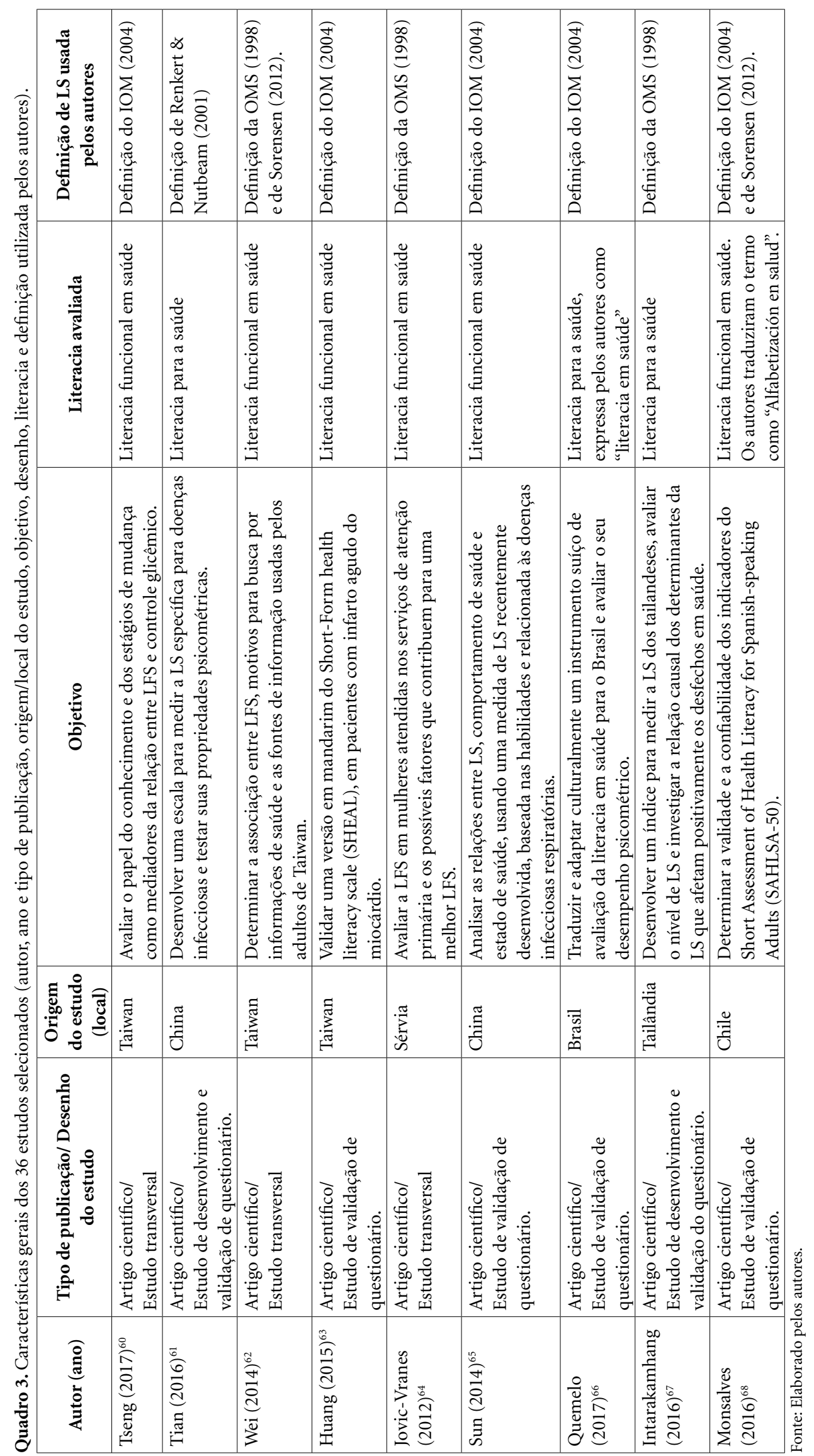




\section{Discussão}

O presente estudo teve como objetivo conduzir uma revisão sistemática dos estudos de avaliação da LS em países menos desenvolvidos, tendo como base a classificação do Banco Mundial para países de economias de renda baixa ou média ${ }^{17}$, e/ ou em países latino-americanos, a fim de se identificar: a definição conceitual considerada pelos autores para o construto da LS; a metodologia empregada e o questionário ou o instrumento de avaliação utilizado; e se há diferenças na forma como os países menos desenvolvidos estão avaliando este construto, comparativamente aos países desenvolvidos, usando como referência a revisão sistemática realizada por Sorensen ${ }^{16}$.

Dos 6.025 estudos captados inicialmente nas buscas realizadas em 5 bases de dados (Medline, Scopus, Embase, LILACS e SciELO), foram selecionados para a amostra final 36 estudos. Destes, a maioria $(58,3 \%)$ era proveniente de países do continente asiático (China, Taiwan, Irã e Tailândia), seguidos pelos estudos provenientes de países da América do Sul, como Brasil, Argentina e Chile (27,8\%). Apenas 11 estudos (11,1\%) eram provenientes de países europeus, como Turquia e Sérvia e 1 estudo era da Oceania $(2,8 \%)$. Da amostra final, os países com maior número de publicações foram: China (25\%), seguida por Taiwan (22,2\%) e Brasil (19,4\%).

Em relação do desenho do estudo, a grande maioria $(97,2 \%)$ envolvia o desenvolvimento, a validação ou a aplicação de algum questionário. Somente um estudo $(2,8 \%)$ descrevia o desenvolvimento de um material educativo, uma cartilha educativa para indivíduos portadores de doença renal crônica. Os questionários mais frequentemente utilizados nos estudos foram: o TOFHLA, em sua versão original ou abreviada, o SAHLSA ou o SAHLPA, o NVS e o REALM. Todos são instrumentos que medem a dimensão funcional ou básica da LS. Também foram observados estudos que utilizavam questionários voltados para populações específicas, áreas temáticas específicas, outras escalas de avaliação deste construto e estudos qualitativos.

Em relação à dimensão da LS que foi abordada, percebe-se, ainda, que a maior parte dos estudos $(50 \%$ dos estudos selecionados nesta revisão) ainda se concentra na dimensão funcional ou básica da LS, a LFS. No entanto, já se observa, na literatura, um esforço no sentido de serem desenvolvidos instrumentos e outras formas de mensuração das demais dimensões da literacia propostas por Nutbeam, ou seja, a LS propria- mente dita, visto que, quase $40 \%$ dos estudos selecionados para a amostra final $(14 ; 38,9 \%)$ avaliaram a LS. Se considerarmos que, dos quatro estudos que tratavam de literacia aplicada a outros domínios mais específicos da saúde, como a odontologia ou a alimentação e nutrição, este último abordou o conceito multidimensional da LS e os outros três trataram da sua primeira dimensão - a LFS -, o percentual de estudos que se concentrou na dimensão funcional ou básica da LS seria de $58,3 \%$, enquanto que o percentual de estudos que conseguiu capturar as demais dimensões da LS seria de $41,7 \%$.

Em relação à definição conceitual considerada pelos autores para o construto da LS, as mais frequentemente citadas nos estudos foram, em ordem decrescente: a definição do IOM, de 2004 $(36,1 \%)^{54}$, a definição da OMS, de $1998(19,4 \%)^{55}$ e a definição do estudo de Sorensen, de 2012 $(13,9 \%)^{16}$. Na revisão sistemática realizada por Sorensen e colaboradores ${ }^{16}$, em países desenvolvidos, as definições para LS mais frequentemente citadas foram as do IOM (2004), da OMS (1998) e da AMA (1999) ${ }^{56}$. No presente estudo, a definição da AMA teve um percentual menor $(5,6 \%)$, comparativamente às demais definições. Provavelmente isto se deveu ao Sorensen ter selecionado mais estudos provenientes dos Estados Unidos, por ter avaliado somente países desenvolvidos, diferentemente do presente estudo, que avaliou apenas países de economias de renda baixa ou média ou países latino-americanos. Ainda assim, verificou-se que, tanto nos países desenvolvidos, como naqueles com economias de rendas baixa ou média, as definições do IOM e da OMS para o construto da LS as mais frequentemente citadas. Além disso, nesta revisão, observou-se que a definição de LS proposta pelo modelo teórico desenvolvido no estudo de Sorensen ${ }^{16}$ também apresentou um grande número de citações.

Observa-se que, em seis estudos $(28,6 \%)$, dos 21 que avaliaram a dimensão funcional da LS, incluindo os que analisaram a LFS no contexto da saúde bucal, os autores descreveram como definições para LS àquelas mais relacionadas à multidimensionalidade deste construto, como as definições de: Sorensen ${ }^{16}$, OMS $^{55}$, Kwan $^{57}$ e Nutbeam ${ }^{2,7}$. Esse percentual é bastante alto e é interessante observar que, mesmo que os autores considerem a multidimensionalidade do construto, ainda é mais fácil ou mais comum na literatura a opção por avaliar a LFS. Além disso, observa-se, nesses estudos de avaliação da LFS, uma carência de discussão sobre as limitações de se captar apenas uma dimensão da LS, principalmente naqueles 
que utilizam como referencial teórico a definição mais abrangente e multidimensional da LS.

Em relação às nomenclaturas utilizadas para LS, dos dez estudos conduzidos em países latino -americanos, que utilizam o idioma português ou espanhol, utilizaram-se as seguintes terminologias para representar o construto da LS ou da LFS: alfabetização em saúde, alfabetismo em saúde, letramento em saúde e literacia em saúde, no Brasil, e alfabetización en salud, nos estudos conduzidos na Argentina e no Chile. Os demais estudos utilizaram o termo em inglês health literacy. Ao considerarmos apenas os estudos conduzidos no Brasil, observa-se que houve certa preferência $(57,1 \%)$ pelo termo letramento em saúde. No entanto, em estudo recentemente publicado (2017), já foi usada a expressão literacia em saúde. Nos outros dois estudos brasileiros foram usados termos semelhantes aos dos demais estudos latino-americanos, que usaram a expressão alfabetização em saúde.

O presente estudo verificou que o maior número de publicações existentes na literatura até o momento sobre LS, em países de economias de renda baixa ou média e em países latino-ameri- canos, é oriunda de países como China, Taiwan e Brasil. No Brasil, o percentual de estudos publicados sobre o tema foi de aproximadamente $20 \%$, bem mais alto que o dos demais países da América do Sul, mostrando um crescente interesse pela temática da LS. Os estudos publicados em países menos desenvolvidos, da mesma forma que o observado na revisão sistemática de Sorensen ${ }^{16}$, utilizaram mais frequentemente as definições para LS do $\mathrm{IOM}^{54}$ e da OMS ${ }^{55}$. A maioria dos estudos publicados $(58,3 \%)$ avalia apenas a LFS, a dimensão funcional ou básica da $\mathrm{LS}^{2}$, mas já há um percentual considerável de estudos $(41,7 \%)$ que vêm buscando captar a multidimensionalidade deste construto. É interessante notar que quase 30\% dos estudos que tiveram como objetivo avaliar a LFS utilizaram como referencial teórico as definições mais abrangentes de LS, indicando que, mesmo considerando a multidimensionalidade do construto, ainda é mais frequente na literatura a opção por avaliar a LFS. Também se observou uma carência de discussão sobre as limitações de se captar apenas uma dimensão da LS, principalmente nos estudos que utilizaram como referencial teórico a definição multidimensional da LS.

\section{Colaboradores}

ALB Pavão trabalhou em todas as etapas de elaboração do artigo, desde a concepção, metodologia, resultados e redação do artigo. GL Werneck trabalhou na concepção, metodologia e redação final. 


\section{Referências}

1. Sørensen K, Pelikan JM, Röthlin F, Ganahl K, Slonska Z, Doyle G, Fullam J, Kondilis B, Agrafiotis D, Uiters E, Falcon M, Mensing M, Tchamov K, van den Broucke S, Brand H; HLS-EU Consortium. Health literacy in Europe: comparative results of the European health literacy syrvey (HLS-EU). European J Public Health 2015; 25(6):1053-1058.

2. Nutbeam D. The evolving concept of health literacy. Soc Sci Med (1982) 2008; 67:2072-2078.

3. Paasche-Orlow MK, Parker RM, Gazmararian JA, Nielsen-Bohlman LT, Rudd RR: The prevalence of limited health literacy. J Gen Intern Med 2005; 20:175184.

4. Berkman ND, Sheridan SL, Donahue KE, Halpern DJ, Crotty K. Low health literacy and health outcomes: an updated systematic review. Ann Intern Med 2011; 155:97-107.

5. Nielsen-Bohlman L, Panzer AM, Kindig DA, editors. Health literacy: a prescription to end confusion. Washington, DC: National Academies Press; 2004.

6. Zarcadoolas C, Pleasant A, Greer D. Advancing health literacy: a framework for understanding and action. San Francisco, CA: Jossey Bass; 2006.

7. Nutbeam D. Advancing health literacy: a global challenge for the 21st century. Health Promot Int 2000; 15:183-184.

8. Pleasant A. Advancing health literacy measurement: a pathway to better health and health system performance. J Health Commun 2014; 19:1481-1496.

9. Baker DW, Parker RM, Williams MV, Clark WS, Nurss $J$. The relationship of patient reading ability to self-reported health and use of health services. Am J Public Health 1997; 87(6):1027-1030.

10. World Health Organization (WHO). Health literacy: the solid facts. Denmark: WHO; 2013. [cited 2019 Apr. 25]. Available from: http://www.euro.who.int/_data/ assets/pdf_file/0008/190655/e96854.pdf

11. Brown H, Prisuta R, Jacobs B, Campbell A. US Department of Education. National Center for Education Statistics. Literacy of Older Adults in America: Results from the National Adult Literacy Survey, NCES 97-576. Washington, DC: 1996, apud Paasche -Orlow MK, Wolf MS: The causal pathways linking health literacy to health outcomes. Am J Health Behav 2007; 31(Supl. 1):19-26.

12. SSchillinger D, Grumbach K, Piette J, Wang F, Osmond D, Daher C, Palacios J, Sullivan GD, Bindman AB. Association of health literacy with diabetes outcomes. JAMA 2002; 288(4):475-482.

13. Martins R, Saboga-Nunes L. The challenges of epistemological validation to Brazil of the European health literacy survey (HLS-EU-BR). Aten Primaria 2014; 46 (Espec. Cong):12.

14. Saboga-Nunes L, Sørensen K, Pelikan J, Cunha M, Rodrigues E, Paixão E. Cross-cultural adaptation and validation to portuguese of the European health literacy survey (HLS-EU-PT). Aten Primaria 2014; 46 (Espec. Cong):13.

15. Martins NFF, Abreu DPG, Silva BT, Semedo DSRC, Pelzer MT, Lenczak FS. Letramento funcional em saúde e adesão à medicação em idosos: revisão integrativa. Rev Bras Enferm 2017; 70(4):868-874.
16. Sørensen K, Van den Broucke S, Fullam J, Doyle G Pelikan J, Slonska Z, Brand H; (HLS-EU) Consortium Health Literacy Project European. Health literacy and public health: a systematic review and integration of definitions and models. BMC Public Health 2012; 25; 12:80.

17. The World Bank [Website]. World Bank Country and Lending Groups. [cited 2017 Jul. 25]. Available from: https://datahelpdesk.worldbank.org/knowledgebase/ articles/906519-world-bank-country-and-lendinggroups

18. Duong TV, Chang PW, Yang SH, Chen MC, Chao WT, Chen T, Chiao P, Huang HL. A new comprehensive short-form health literacy survey tool for patients in general. Asian Nurs Res 2017; 11:30-35.

19. Cordeiro MD, Arruda SPM, Lima PPS, Reis DM, Mendes RCM, Mendonça MP, Sampaio HAC. Associações entre letramento em saúde bucal, consumo alimentar e presença de lesões orais. Nutr Clin Diet Hosp 2017; 37(1):49-56.

20. Santos JEM, Brasil VV, Moraes KL, Cordeiro JABL, Oliveira GF, Bernardes CP, Bueno BRM, Boaventura RP, Gonçalves FAF, Oliveira LMAC, Barbosa MA, Silva AMTC. Legibilidade de prospecto facilitador e letramento em saúde de indivíduos com marcapasso. Rev Bras Enferm 2017; 70(3): 661-667.

21. Liao LL, Liu CH, Cheng CC, Chang TC. Defining Taiwanese children's health literacy abilities from a health promotion perspective. Glob Health Promot 2017; 24(4):69-80

22. Sun X, Shi Y, Zeng Q, Wang Y, Du W, Wei N, Xie R, Chang C. Determinants of health literacy and health behavior regarding infectious respiratory diseases: a pathway model. BMC Public Health 2013; 13:261.

23. Doustmohammadian A, Omidvar N, KeshavarzMohammadi N, Abdollahi M, Amini M, Eini-Zinab $\mathrm{H}$. Developing and validating a scale to measure Food and Nutrition Literacy (FNLIT) in elementary school children in Iran. PLoS ONE 2017; 12(6):e0179196.

24. Santos LTM, Bastos MG. Desenvolvimento de material educacional sobre doença renal crônica utilizando as melhores práticas em letramento em saúde. J Bras Nefrol 2017; 39(1):55-58.

25. Leung AY, Cheung MK, Lou VW, Chan FH, Ho CK, Do TL, Chan SS, Chi I. Development and validation of Chinese health literacy scale for diabetes. J Clinical Nurs 2012; 22:2090-2099.

26. Wong HM, Bridges SM, Yiu CKY, McGrath CPJ, Au TK, Parthasarathy DS. Development and validation of Hong Kong rapid estimate of adult literacy in dentristy. J Investig Clin Dent 2012; 3:118-127.

27. Chung MH, Chen LK, Peng LN, Chi MJ. Development and validation of the health literacy assessment tool for older people in Taiwan: potential impacts of cultural differences. Arch Gerontol Geriatr 2015; 61:289295.

28. Bridges SM, Parthasarathy DS, Au TKF, Wong HM, Yiu CKY, McGrath CPJ. Development of functional health literacy assessment instruments: application of literacy and cognitive theories. J Public Health Dent 2014; 74:110-119. 
29. Pan FC, Su CL, Chen CH. Development of a health literacy scale for Chinese-speaking adults in Taiwan. Int J Health Medical Engin 2010; 4(1):29-35.

30. Khajouei R, Salehi F. Health literacy among Iranian high school students. Am J Health Behav 2017; 41(2):215-222.

31. Yilmazel G, Cetinkaya F. Health literacy among schoolteachers in Çorum, Turkey. East Mediterr Health J 2015; 21(8):598-605.

32. Wang C, Li H, Li L, Xu D, Kane RL, Meng Q. Health literacy and ethnic disparities in health-related quality of life among rural women: results from a Chinese poor minority area. Health Quality Life Outcomes 2013; 11:153.

33. Jovic-Vranes A, Bjegovic-Mikanovic V, Marinkovic J, Kocev N. Health literacy in a population of primary health-care patients in Belgrade, Serbia. Int J Public Health 2011; 56:201-207.

34. Konfino J, Mejia R, Majdalani MP, Perez-Stable EJ. Alfabetizacion en salud en pacientes que asisten a un hospital universitário. Medicina (B Aires) 2009; 69(6):631-634.

35. Keles OR, Ilicali NI, Ozcan MC, Topsever P. Health literacy in patients with type 2 diabetes and its relationship with glycemic control and sociodemographic factors: preliminary results of a descriptive study. Primary Care Diabetes 2013; 1-89 (Espec.Cong):81-82.

36. Sampaio HAC, Carioca AAF, Sabry MOD, Santos PM, Coelho MAM, Passamai MPB. Letramento em saúde de diabéticos tipo 2: fatores associados e controle glicêmico. Cien Saude Colet 2015; 20(3):865-874.

37. Ghanbari S, Ramezankhani A, Montazeri A, Mehrabi Y. Health literacy measure for adolescentes (HELMA): development and psychometric properties. PLoS ONE 2016; 11(2):e0149202.

38. Paskulin LMG, Bierhals CCBK, Valer DB, Aires M, Guimarães NV, Brocker AR, Lanziotti LH, Morais EP. Alfabetização em saúde de pessoas idosas na atenção básica. Acta Paul Enferm 2012; 25 (n. esp. 1): 129-135.

39. Bohanny W, Wu SFV, Liu CY, Yeh SH, Tsay SL, Wang TJ. Health literacy, self-efficacy, and self-care behaviors in patients with type 2 diabetes mellitus. $J$ Am Assoc Nurse Pract 2013; 25:495-502.

40. Bolivar AA, Lanteri ME, Cícero CY, Pérez A, Puchulu FM, Mejía R. Alfabetización en salud y control de la diabetes en pacientes de un hospital universitario de Argentina. Medicina (Buenos Aires) 2017; 77:167-172.

41. Wang X, Guo H, Wang L, Li X, Huang M, Liu Z, Liu $\mathrm{X}$, Wang K, Alamian A, Anderson JL. Investigation of residents' health literacy status and its risk factors in Jiangsu Province of China. Asia Pac J Public Health 2015; 27(2): NP2764-NP2772.

42. Lam LT, Yang L. Is low health literacy associated with overweight and obesity in adolescents: an epidemiology study in a 12-16 years old population, Nanning, China, 2012. Arch Public Health 2014; 72:11.

43. Lee YJ, Shin SJ, Wang RH, Lin KD, Lee YL, Wang YH. Pathways of empowerment perceptions, health literacy, self-efficacy, and self-care behaviors to glycemic control in patients with type 2 diabetes mellitus. $\mathrm{Pa}$ tient Educ Cous 2016; 99:287-294.

44. Apolinario D, Braga RCOP, Magaldi RM, Busse AL, Campora F, Brucki S, Lee SY. Short Assessment of health literacy for portuguese-speaking adults. Rev Saude Publica 2012; 46(4):702-711.
45. Tseng HM, Liao SF, Wen YP, Chuang YJ. Stages of change concepto $f$ the transtheoretical model for healthy eating links health literacy and diabetes knowledge to glycemic control in people with type 2 diabetes. Primary Care Diabetes 2017; 2:29-37.

46. Tian X, Di Z, Cheng Y, Ren X, Chai Y, Ding F, Chen J, Southerland JL, Cui Z, Hu X, Xu J, Xu S, Qian G, Wang L. Study in the development of an infectious disease-specific health literacy scale in the Chinese population. BMJ Open 2016; 6:e012039.

47. Wei MH. The associations between health literacy, reasons for seeking health information, and information sources utilized by Taiwanese adults. Health Educ J 2014; 73(4):423-434.

48. Huang YJ, Wang YL, Wu TY, Chen CT, Kuo KN, Chen SS, Hou WH, Hsieh CL. Validation of the short-form health literacy scale in patients with stroke. Patient Educ Cous 2015; 98:762-770.

49. Jovic-Vranes A, Bjegovic-Mikanovic V. Which women patients have better health literacy in Serbia? Patient Educ Cous 2012; 89:209-212.

50. Sun X, Yang S, Fisher EB, Shi Y, Wang Y, Zeng Q, Ji Y, Chang C, Du W. Relationships of health literacy, health behavior, and health status regarding infectious respiratory diseases: application of a skill-based measure. J Health Commun 2014; 19:173-189.

51. Quemelo PRV, Milani D, Bento VF, Vieira ER, Zaia JE. Literacia em saúde: tradução e validação de instrumento para pesquisa em promoção da saúde no Brasil. Cad Saude Publica 2017; 33(2):e00179715.

52. Intarakamhang $\mathrm{U}$, Kwanchuen $\mathrm{Y}$. The development and application of the ABCDE-health literacy scale for Thais. Asian Biomedicine 2016; 10(6):587-594.

53. Monsalves MJ, Mañalich J, Fuentes E. Validación del test Short Assessment of Health Literacy for Spanish -Speaking Adults en Chile, para medir alfabetización en salud. Rev Med Chile 2016; 144:604-610.

54. Institute of Medicine (IOM): Health literacy: a prescription to end confusion. Washington, DC: The National Academies; 2004.

55. Nutbeam D: Health Promotion Glossary. Health Promot Int 1998; 13:349-364.

56. Ad Hoc Committee on Health Literacy for the Council on Scientific Affairs AMA: Health literacy: report of the council on scientific affairs. J Am Med Assoc 1999; 281(6):552-557.

57. Kwan B, Frankish J, Rootman I. The development and validation of measures of "health literacy" in different populations. Columbia: University of British Columbia, Institute of Health Promotion Research; 2006.

58. Parker R, Baker D, Williams M, Nurss J. The test of functional health literacy in adults: A new instrument for measuring patients' health literacy. J Gen Int Med 1995; 10:537-541.

Artigo apresentado em 04/05/2019

Aprovado em 19/07/2020

Versão final apresentada em 21/07/2020

Editores-chefes: Romeu Gomes, Antônio Augusto Moura da Silva 